\title{
Perceived orientation, motion, and configuration of the body during viewing of an off-vertical, rotating surface
}

\author{
PAUL A. DiZIO and JAMES R. LACKNER \\ Brandeis University, Waltham, Massachusetts
}

\begin{abstract}
Dynamic patterns of activity from multiple receptor systems, as well as efferent signals associated with voluntary movements, influence perceived body motion. The experiment to be described explored how these factors interrelate in influencing apparent body motion. It involved exposing stationary, reclining subjects to a patterned surface which rotated around a vertical or an off-vertical axis. We were able to create situations in which the combined patterns of visual, otolithic, somatosensory, and semicircular canal stimulation actually present were not consistent with body motion in a terrestrial environment. Nevertheless, all of our subjects experienced self-rotation and displacement around a vertical axis. A variety of changes in apparent body orientation, body configuration, and slant of the visual surface occurred concurrently with the elicitation of apparent body rotation. These perceptual remappings were such as to be consistent both with the rotary visual stimulation present and with the absence of changes in otolithic and somatosensory inputs. They were achieved through a reinterpretation of the static otolithic, somatosensory, and proprioceptive signals present. Our findings demonstrate that perceived body motion also depends on representations of what combinations of sensory input are possible in a terrestrial environment.
\end{abstract}

The control and appreciation of body orientation are related to the activity of multiple receptor systems. Changing patterns of visual, auditory, vestibular, proprioceptive, surface and deep somatosensory stimulation, as well as efferent activity associated with voluntary movement, can all influence apparent body attitude, configuration, and motion. For example, the following types of stimulation can make stationary individuals feel apparent body motion: an optokinetic field (Dichgans \& Brandt, 1978; Mach, 1959), a rotating sound-field (Dodge, 1923; Lackner, 1977), contact of a rotating surface with the soles of the immobilized feet (Lackner \& DiZio, 1984), passive following of a moving substrate with the arms or legs (Brandt, Buchele, \& Arnold, 1977; Lackner \& DiZio, 1984), active stepping in place on a motor driven floor while holding an inertially stable bar (Bles, 1981; Bles \& Kapteyn, 1977), pedaling of a freewheeling platform while sitting on a fixed chair (Lackner \& DiZio, 1984), and vibration of postural muscles to elicit reflex contractions while the body is restrained (Lackner \& Levine, 1979).

A possible physiological basis for such multisensory influences on perceived motion and orientation has been discovered in recent years. Cells have been identified in the

Support was provided by National Aeronautics and Space Administration contract NAS 9-15147. The authors' mailing address is: Ashton Graybiel Spatial Orientation Laboratory, Brandeis University, Waltham, MA 02254 . vestibular nuclei whose discharge rates are influenced by both body motion, as signaled by the vestibular end organs, and visual motion (Allum, Graf, Dichgans, \& Schmidt, 1976; Azzena, Azzena, \& Marini, 1974; Henn, Young, \& Finley, 1974; Waespe \& Henn, 1977). Convergences of vision, audition, somatosensation, and proprioception at the cellular level in the vestibular nuclei have been demonstrated (Daunton \& Thomsen, 1979; Duensing \& Schaefer, 1958; Rubin, Liedgren, Milne, Young, \& Fredrickson, 1977; Thoden, Golsong, \& Wirbitzky, 1975; Wilson, Kato, Thomas, \& Peterson, 1966). Similarities between perceived self-motion and the dynamic responses of nodal vestibular nuclei cells to multiple sensory inputs have motivated analogies between perceptual and physiological levels of description (Henn, Cohen, \& Young, 1980). Mathematical models of perception based on sensory convergence of peripheral receptive fields onto single neurons, whether they include linear or nonlinear interactions, will be referred to here as convergence models. Currently, the most sophisticated of these models concern visual and semicircular canal convergences.

Sensory convergence models of perceived body motion (e.g., Henn et al., 1980; Mergner, Nardi, Becker, \& Deecke, 1983) are supported by the presence of neural substrates in the limited sense that different sensory afferents do, in fact, meet within the nervous system. Although both visual and vestibular stimulation influence the same vestibular nuclei cells, the paths they take are 
very different. The dynamic response of the semicircular canal afferents to angular acceleration is generally agreed upon, as is the nature of the projections through the eighth nerve directly to the site of interaction. Thus, the path is direct and the analogous elements in mathematical convergence models correspond to accepted neuronal operations. The visual pathway is more complex. There is no consensus about the neural mechanism for extracting motion information dimensionally compatible with the vestibular contribution or about the anatomical connection to the vestibular nuclei. In addition, the same populations of cells whose output is a function of visualvestibular interaction are the target of other sources of sensory input, such as somatosensory and proprioceptive, as cited above. Little is known about whether neural integration occurs before the points of convergence. Thus, weighting of the different sensory inputs prior to the nodal points, hypothesized by Zacharias and Young (1981), is a complex sort of integration that has no known neural basis.

In fact, this problem of how to interrelate the inputs with the perceptual output is central to any model of perceived body motion. Input combinations concordant with physical states must be mapped with corresponding phenomenal states. Such combinations can be dynamic. For example, when a person undergoes angular acceleration in a structured visual field, the semicircular canals are stimulated, as are the visual motion detectors. Thus, a model should specify both sources as having impact upon a summation point whose output is proportional to body motion. After the body reaches constant angular velocity, the canal response decays but the visual response does not. Because the individual continues to feel the same rotation, the visual input must be weighted more heavily. The model of Zacharias and Young (1981) represents a nonlinearity of this kind by an element that measures cue conflict, and the relative weighting of visual and vestibular inputs depends on whether they conflict (as they do during prolonged constant velocity rotation) or not (as during acceleration). This nonlinearity enables the model to predict the time course of cessation of perceived selfmotion during prolonged, constant velocity body rotation in the dark and also of visually induced, illusory selfrotation (ISR).

Extensive testing of convergence models of perceived body motion has been conducted using prolonged, constant-velocity rotation around the vertical $z$-axis of the body or about the vertical x-axis of the head (Bles \& Kapteyn, 1977; Dichgans \& Brandt, 1972; Grusser \& Grusser-Cornehls, 1973; Huang \& Young, 1981; Mergner et al., 1983; Waespe, Waespe, \& Henn, 1980; Young, Dichgans, Murphy, \& Brandt, 1973).

In our experiment, cue conflict originated not just from unnatural stimulation, as in the case of visually induced ISR about a vertical axis, but also from stimulation that can never exist during body motion in a terrestrial environment. This was achieved by exposing supine subjects to a radial pattern upon a large disk rotating about vertical and off-vertical axes. There were four viewing conditions, each involving different combinations of head and eye position with respect to gravity and to the axis of disk rotation.

In two of the conditions, the disk was rotated about a vertical axis with the subject looking straight up at its center of rotation, a situation in which the visual, canal, otolithic, and somatosensory activity patterns are consistent with self-rotation about the optic axis or with visual motion about the optic axis (see Figure 1a). By contrast, in the other two viewing conditions, the visual display, along with its axis of rotation, was tilted by $30^{\circ}$. This is a situation in which the visual and canal activity is consistent with either body rotation or visual rotation but the otolithic and somatosensory cues are compatible only with visual rotation. This is so in the latter case because if the
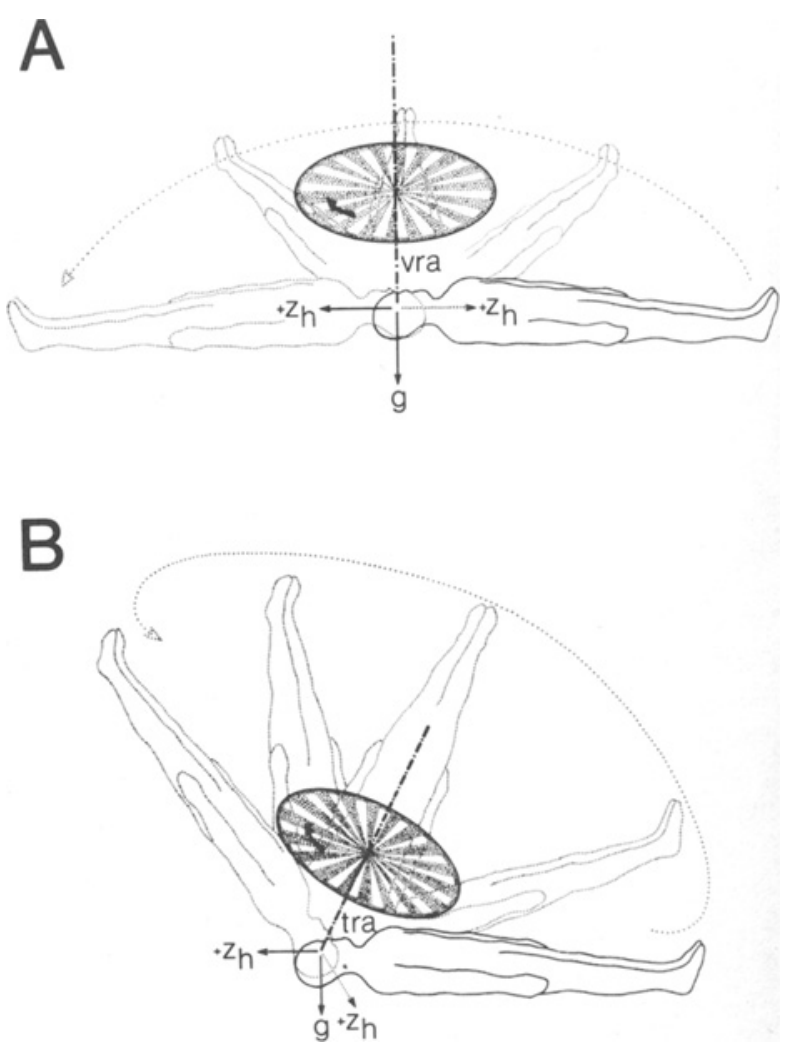

Figure 1. When a physically stationary, supine observer views a rotating disk with a radial pattern on its surface (stipple pattern and solid arrow), the visual stimulation is also consistent with perception of a stationary disk and body rotation around the same axis in the opposite direction (dotted arrow). Panel $A$ shows a series of body positions (dotted figures) consistent with such visually induced illusory body motion around a vertical rotation axis (vra). Panel B shows it for a titled rotation axis (tra). If the body were actually moving in the illusory pattern shown, the angle between $+z$-axis of the head $(+\mathrm{zh})$ and the gravitational force vector $(\mathrm{g})$ would change continuously in (B), leading to changing patterns of otolithic and somatosensory stimulation inconsistent with those static cues actually present in the stationary observer, but not in $(A)$, where the patterns would remain consistent with the cues actually present. 
body were rotating about the tilted optic axis, as in Figure $1 b$, the otolithic organs, which are sensitive to gravitoinertial force, would signal constant reorientation of the body with respect to the gravitational vertical. The absence of such a dynamic pattern of otolithic activity is inconsistent with body rotation around an off-vertical axis on Earth. The absence of a continuously rotating shear force vector stimulating somatosensory receptors in the surface and deep tissues of the body is also inconsistent with rotation of the body about an off-vertical axis.

We expected that illusory body motion and displacement would be induced during the viewing of a surface rotating about an off-vertical axis only if the rotation axis were reinterpreted as being vertical. We expected that if this happened, other changes in body orientation, compatible with the reinterpreted axis of visual rotation, would occur. The nature of these changes allows one to probe the way mechanisms subserving perceived body motion organize peripheral inputs. On the other hand, no clear predictions can be derived from existing convergence models. It is impossible, for example, for otolithic and somatosensory inputs to merge directly with canal and visual inputs because they are dimensionally incompatible. The otoliths provide metrics of tilt or linear acceleration, and the canals ordinarily provide metrics of angular velocity. It is possible that convergence models could be modified so that otolithic and somatosensory inputs would influence the relative weighting of canal and visual channels, but such an arrangement could only affect the resultant angular velocity. Convergence models also make no predictions about the perceived axis of rotation, nor do they aid in formulating hypotheses about what additional factors might be involved in judging body orientation.

Illusory self-rotation around a vertical axis was induced in all of our conditions. Moreover, in the conditions in which the rotation axis was off-vertical, the other expected perceptual changes occurred simultaneously. There were shifts in apparent orientation of the visual surface, the head, eyes, torso, or the entire body with respect to the subjective vertical. In some of these instances, apparent eye-head and head-trunk configurations were altered. Illusory self-rotation was abolished along with the other perceptual changes that were present if head or eye movements were made. We will demonstrate that these findings show that the appreciation of body motion, attitude, and configuration depend on levels of representation and interacting computations which are exclusive of those implicated by convergence models.

\section{METHOD}

\section{Subjects}

Twelve individuals with normal or corrected vision and without a history of sensorimotor anomaly participated. Nine were ignorant of the objectives of the experiment.

\section{Apparatus}

A low horizontal cart on which the subject lay supine was rolled along a track to position the individual beneath the visual display.
An air mattress supported the subject's body. The angle of the subject's head in relation to the trunk and to the gravitational vertical was adjusted and maintained with a foam rubber support. A motordriven disk, $100 \mathrm{~cm}$ in diameter, was positioned $50 \mathrm{~cm}$ from the subject's face and subtended approximately $90^{\circ}$ of his or her visual field. The surface of this disk contained alternating black and white stripes converging toward the center. The disk was mounted at its center on a shaft that could be tilted fore-aft with respect to the subject's head. A black curtain on a frame formed a tent around the disk and the upper part of the subject's body. Within this test chamber, incandescent lights were used to maintain an average luminance of $0.9 \mathrm{fL}$. At this scotopic level, a subject could identify features of the radial pattern, but not the details of the curtained chamber, his or her body, or the edges of the disk while fixating its center. Accordingly, there was no visual frame of reference other than the radial pattern. Figure 2 illustrates the apparatus.

\section{Procedure}

Each subject participated on a single test day in the four conditions illustrated in Figure 3. In two of the conditions, the disk was horizontal; in the other two, it was tilted $30^{\circ}$ from the horizontal. Within these conditions, the subject's head and eye positions were different. The configurations included: (A) disk horizontal, head horizontal; (B) disk and head parallel and tilted $30^{\circ}$ up in relation to the horizontal; (C) disk tilted $30^{\circ}$ up in relation to the horizontal, head horizontal; and (D) disk horizontal, head back $30^{\circ}$. All subjects were tested once in each of the four conditions. Condition A, the canonical, or baseline, condition, was always first, and the sequence of the remaining conditions was counterbalanced across subjects.

During all trials, the subject was positioned so that his or her line of sight coincided with the axis of disk rotation. The subject was instructed to maintain fixation of the center of the visual display, so there was a $30^{\circ}$ depression of gaze in Conditions B and D. The disk was turned either clockwise or counterclockwise at $48 \% \mathrm{sec}$ for $3 \mathrm{~min}$, stopped and held stationary for $1 \mathrm{~min}$, and then rotated in the opposite direction at $48^{\circ} / \mathrm{sec}$ for $3 \mathrm{~min}$ and stopped. In a pilot study, $48 \% \mathrm{sec}$ was in the middle of the range of speeds at which most subjects, when tested in the baseline condition, came to report self-motion and minimal disk motion. It was also found that induced body motion and the attendant illusory changes in orientation waned after about $3 \mathrm{~min}$, but returned to original strength if the disk's direction of rotation was reversed. Accordingly, in the

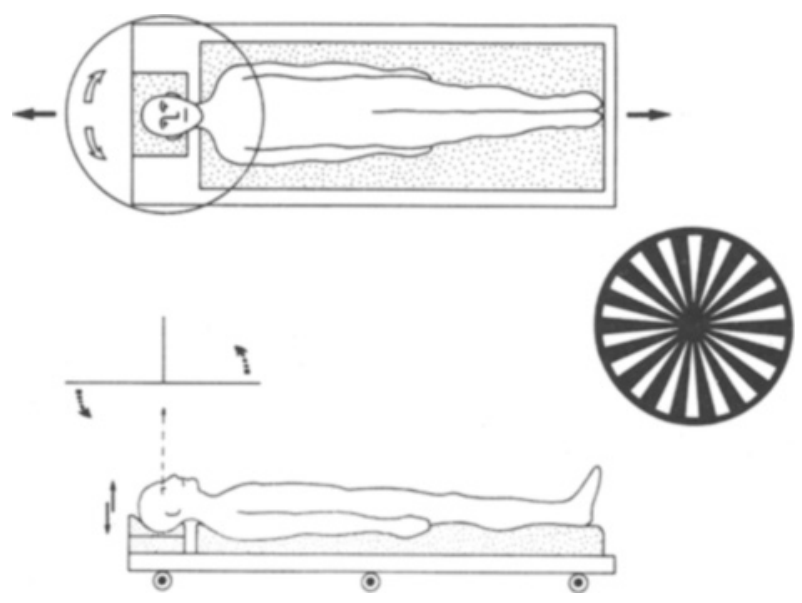

Figure 2. Illustration of the subject in the experimental apparatus. Solid arrows show the directions in which the subject's body could be adjusted. Broken arrows indicate the direction in which the disk angle could be adjusted, and open arrows point to the possible directions of disk motion during visual stimulation. The inset at the right is a scaled likeness of the radial pattern. 

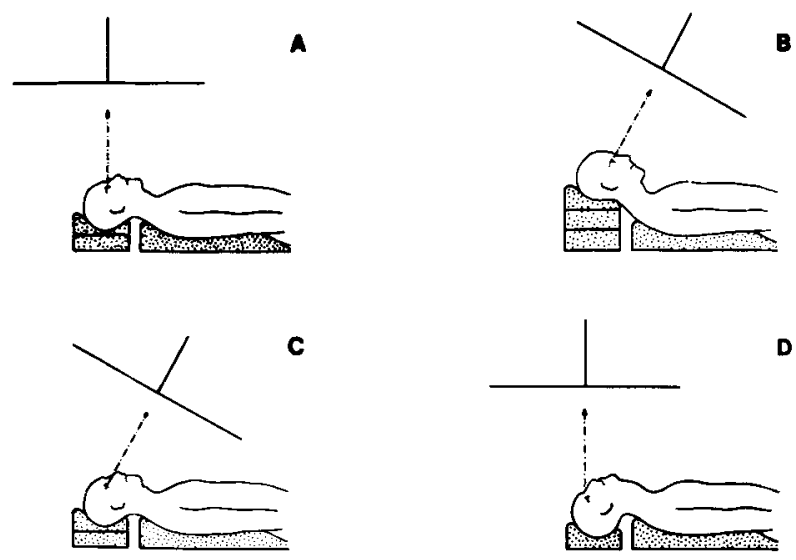

c

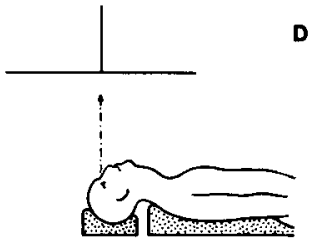

Figure 3. Illustration of the experimental conditions. (A) Disk and head horizontal, (B) disk and head parallel, both tilted $30^{\circ}$ up from the horizontal, (C) head horizontal, disk tilted up $30^{\circ}$, and (D) disk horizontal, head tilted $30^{\circ}$ back in relation to the horizontal. The arrow represents the direction of gaze, which is coaxial with disk rotation in all conditions.

present study, we reversed the direction of disk rotation after $3 \mathrm{~min}$ so that subjects would have increased time to experience the illusory patterns.

During each condition, the subject was required to signal verbally when he or she experienced apparent motion and when changes in his or her own apparent orientation took place; the subject was also required to report changes in the apparent motion and orientation of the disk. The instructions were to report apparent orientation with respect to the subjective gravitational vertical. A tape recorder captured the voices of the subject and experimenter throughout each experimental condition.

Twice during each condition, when a subject was experiencing strong ISR, the experimenter asked him or her to make movements that had been prepracticed. One type of movement involved the subject's swiveling the head side to side at $1.5 \mathrm{~Hz}$ for five cycles while continuing to gaze at the center of the visual pattern. The other involved his or her moving the eyes back and forth from one edge of the visual array to the other at $1.5 \mathrm{~Hz}$ while keeping the head still. The subject reported how these movements affected the apparent movement and orientation of his or her body and of the display and whether they evoked symptoms of motion sickness. In a posttrial interview, the subject helped clarify any of his or her recorded statements that were ambiguous.

\section{RESULTS}

In all conditions, $10-20 \mathrm{sec}$ after the disk began to move, subjects reported ISR in the opposite direction and diminished apparent motion of the visual display. In each condition, the subjects always reported experiencing smooth constant-velocity body rotation about a vertical axis.

In Condition A, the subjects experienced ISR around a vertical axis coupled with a decrease in the velocity of apparent disk motion. By contrast, in Conditions B, C, and $D$, although ISR about a vertical axis was also experienced, various other perceptual changes were also reported. Figure 4 summarizes these changes for the different conditions.
In Condition B, when ISR commenced, all the subjects but one reported that the disk seemed to change position and become horizontal and directly overhead. Seven of the subjects also felt that their heads remained parallel to the disk either owing to apparent dorsiflexion of the neck (J.D., M.O., D.E., J.M., M.D.) or because of apparent declination of the entire head and torso by an equal amount (P.D., C.M.). Three others (J.L., M.L., N.H.) felt changes in both neck angle and body declination, so that the entire body seemed to be resting on the occiput with the feet elevated. Subject E.A. experienced a change in disk orientation without a change in head orientation, but reported a change in the direction of gaze. Subject D.S. experienced ISR around a vertical axis and correctly reported the true orientation of her body and the disk in Condition B and all remaining conditions.

Similar patterns were reported in Condition $C$. Ten subjects perceived themselves as gazing vertically at the center of the disk, which appeared to have tipped toward the horizontal. Four (M.L., J.M., M.D., M.O.) reported only this change, but six (P.D., J.D., E.A., J.L., D.E., N.H.) said it felt as if their entire bodies had tilted with the disk so that they were resting on their occiputs with their feet elevated. One subject (C.M.) reported the above change in body slant and also that the disk had tilted from its original position to beyond the horizontal in the other direction so that it was parallel with his apparently inclined head and torso.

In 21 of the 24 trials in Conditions B and C, subjects reported seeing the disk change orientation and move to a horizontal or near-horizontal position, directly overhead. Sixteen of these trials involved changes in apparent head position or in both head and torso positions consistent, in different degrees, with the perceived axis of disk and body rotation and the apparent horizontal orientation of the disk. In eight cases, there were equivalent changes in both head and torso orientations so that there were no changes in their relationships to the disk. There were five trials with reports of changes in the orientation of the head but not the body; they also included errant reports of headtorso configuration, but the angle between the head and disk was reported correctly. In three trials, subjects experienced more change in head than in torso orientation; here the head-torso configuration and the eye-head position were misperceived, but the true angle between the torso and disk was preserved perceptually. In the five trials where no changes in head or torso orientation were felt in conjunction with shifted disk orientation, a change in apparent eye-head position was reported.

In Condition D, after the onset of ISR, nine subjects reported changes in head, torso, or disk position, or some combination thereof. In this condition, all subjects mentioned that with their bodies supine and necks dorsiflexed they were uncomfortable and felt considerable tension in neck muscles as well as pressure on the back of their heads and necks. Those who reported changes in body orientation also stressed the feeling of pressure on the back of 


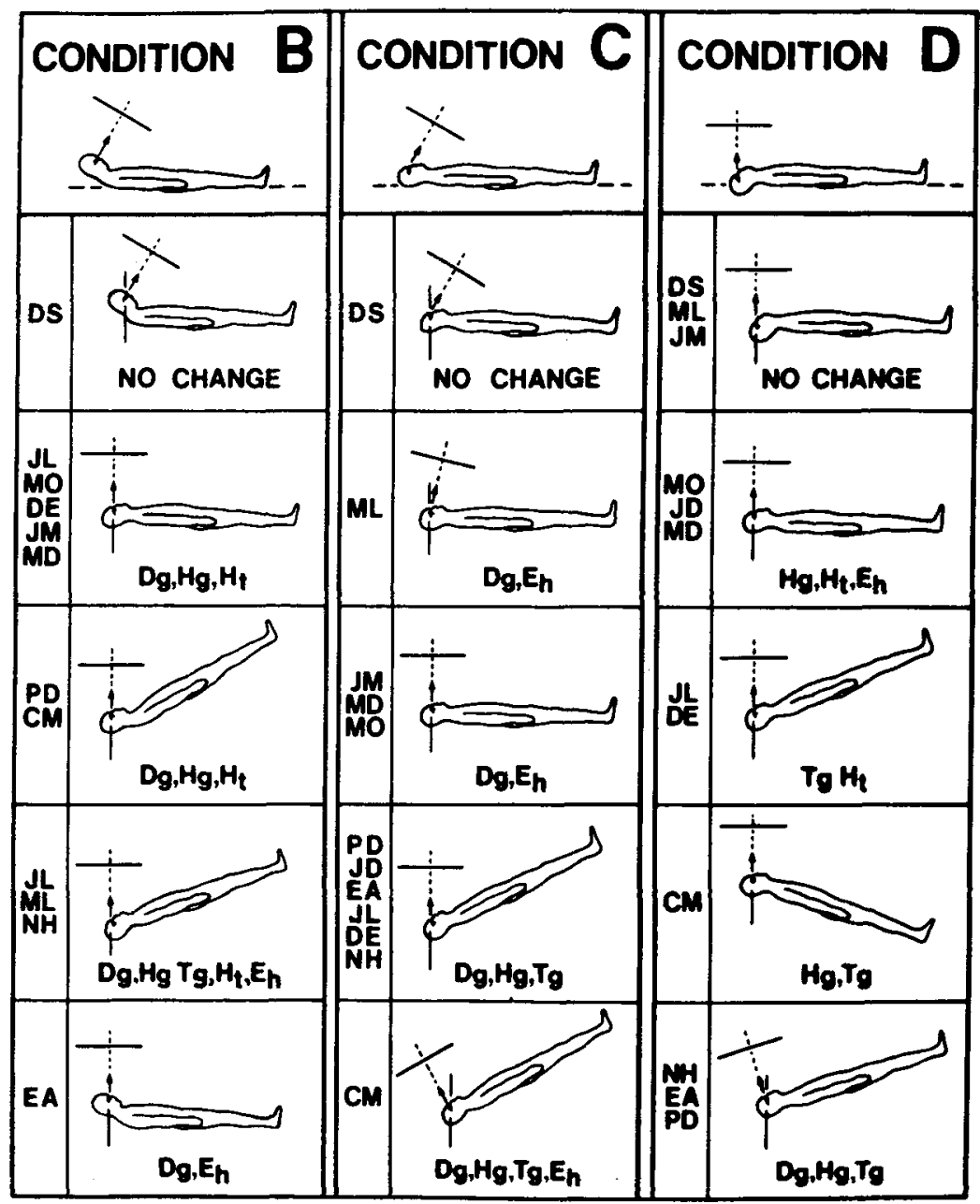

Figure 4. Summary of experienced changes in Conditions $B, C$, and D. The true orientations of subject and disk are shown at the top of each column. The experienced orientations are summarized below with reported head, torso, and disk positions shown in heavy lines; solid lines through the head specify the axis of apparent body rotation, and arrows indicate the apparent line of sight. The initials of the subjects who reported each pattern of orientation are given in the column at the left for each condition. $\mathrm{Dg}, \mathrm{Hg}$, and $\mathrm{Tg}$ indicate the presence of perceived changes in disk, head, and torso orientation with respect to the subjective vertical; Ht and Eh indicate, respectively, changes in apparent head-torso configuration and eye-head position.

the head and stated that the rest of the body seemed to be supported or suspended by the head and neck.

Illusory self-rotation, once elicited, continued in all four conditions throughout the period of disk rotation except when the subjects, upon request, moved their heads or eyes. Movements of either the head or eyes suppressed ISR and restored the correct perception of both body orientation and configuration as well as disk orientation. Table 1 presents the suppression frequencies obtained in each condition expressed as proportions of 12 trials. Ninety-five percent confidence intervals for small-sample proportions computed for each condition never included zero, showing that both eye and head movements had a significant suppressive effect.
Whenever subjects experienced continuous ISR, it felt to them as if their feet were describing a circular path. Many subjects made the unsolicited report that they had experienced patterns of force and airflow on their legs such as would have been present if they had actually been rotating.

Symptoms of motion sickness were not experienced by any of the subjects during either head or eye movements.

\section{DISCUSSION}

The experimental findings provide further insight into the factors that influence perceived body orientation. In 
Table 1

Frequency and Proportion of Suppression of Illusory Self-Rotation by Eye Movements and Head Movements

\begin{tabular}{cccc}
\hline Type of Movement & Condition & Frequency & Proportion \\
\hline Eye & A & 6 & 0.500 \\
& B & 7 & 0.583 \\
& C & 9 & 0.750 \\
Head & D & 7 & 0.583 \\
& A & 10 & 0.833 \\
& B & 11 & 0.917 \\
& C & 9 & 0.750 \\
& D & 9 & 0.750 \\
\hline
\end{tabular}

Note $-n=12 /$ condition

all of the experimental conditions, subjects came to experience ISR about an apparently vertical axis. In Conditions $B$ and $C$, the onset of ISR was associated with changes in the experienced configuration of the body and the apparent axis of rotation of the visual display. It is important to note that in these two conditions, which involved viewing of the off-horizontal disk, the actual patterns of semicircular canal, otolithic, visual, and somatosensory stimulation physically present were not compatible with rotation of the body about the optic axis in a terrestrial environment. The visual cues present were consistent with body rotation around the line of sight; but the otolithic and somatosensory cues were consistent only with self-rotation around a vertical axis. Nevertheless, continuous rotation and displacement of the body were felt by all those tested, and the axis of visually induced ISR coincided with the apparent disk rotation axis and the line of sight. However, the orientations of these axes were misperceived throughout the duration of ISR in Conditions $B$ and $C$ by all but one of the subjects. The axes were actually tilted but were generally perceived as vertical. There were conforming alterations of experienced eye-head and disk-head angles in some cases. In other cases, these changes were not present, but the entire body was perceived to have tilted with respect to gravity just as much as the disk seemed to have. In still other cases, the apparent orientation of either the head or the torso changed so that neck angle was misperceived by an amount equal to the change in disk angle.

Each of these perceptual remappings was consistent with the lack of continuous modulation of both otolithic and somatosensory inputs and with the pattern of dynamic visual stimulation actually present. The perceptual system responded both to the patterns of stimulation present and to the absence of those that would have been present if the body were physically moving by generating combinations of perceived body motion, orientation, and configuration that were possible in a terrestrial environment. However, this was achieved by generating perceptual combinations that were inconsistent with the levels of peripheral otolithic, somatosensory, oculomotor, and proprioceptive activity present.

In Conditions $A$ and $D$, the patterns of peripheral stimulation present were, in fact, consistent with body rotation about a vertical axis in a terrestrial environment, and, in both cases, ISR about a vertical axis was perceived. In Condition A, induction of ISR was not accompanied by other orientational changes; however, in Condition D, limited changes occurred in apparent body orientation but these changes were consistent with the absence of dynamic otolithic and somatosensory activity. As in Conditions B and $C$, the changes were achieved at the expense of disregarding or reinterpreting peripheral otolithic, somatosensory, proprioceptive, or oculomotor signals.

Investigators who test subjects in situations in which all the cues present are consistent only with body rotation about a vertical axis tend to underestimate the complexity of the factors determining spatial orientation. For example, on the basis solely of Condition A in the present experiment, there would be no reason to propose an influence of otolithic and somatosensory cues on visually induced ISR because a model invoking physiological convergence of canal-visual afference at the level of the vestibular nuclei is adequate to explain ISR around the axis of the disk. In fact, most testing of visual-vestibular convergence models has been restricted to such cuecompatible conditions. However, by testing subjects in conditions in which the overall pattern of sensory input is inconsistent with real self-motion, we have shown that perception of body motion must involve virtually all of the body's sensory systems, concurrent analyses of body motion, orientation, and configuration, and rules about what combinations are possible in a terrestrial environment.

There are several investigations relevant to these issues which have led to amendments of the formulation of Zacharias and Young (1981). Held, Dichgans, and Bauer (1975) had vertical subjects view a disk rotating in their frontal planes. The subjects reported continuous body motion in roll and a small body displacement in roll. Because apparent displacement in roll was limited, these investigators hypothesized that a conflict between otolithic inputs, signaling a stationary body, and visual-canal information, signaling body motion in roll, restricted the amount of tilt evoked by the visual-canal convergence alone. In another related experiment, Young, Oman, and Dichgans (1975) positioned observers with their heads $25^{\circ}$ forward, erect, right ear down, or inverted, and exposed them to whole-field visual motion in pitch or roll. They reasoned that the above series of head orientations placed the otoliths in increasingly less sensitive positions (cf. Graybiel \& Patterson, 1954), thereby resulting in a cueconflict signal that would be progressively less effective in attenuating the visual induction of apparent body tilt. The expected pattern of increasing apparent body tilt was obtained and used as evidence to support the existence of sensory convergence and cue-conflict mechanisms as general models of orientation control.

These studies escape the shortcoming mentioned above of providing only concordant sensory cues, but they are limited by the opposite problem: they provided too much discordance. The experiments involve visual field rotation in the frontal plane of upright subjects; consequently, 
a $90^{\circ}$ remapping would have been necessary in order for complete body rotation around the visual axis to have been perceived, given the absence of dynamic otolithic cues. That is, it would have been necessary for the subject to have perceived himself or herself and the visual surface as horizontal. This did not occur, nor did the kind of covariant perceptual changes that we have observed in our test situations. Accordingly, these investigators did not find it necessary to propose the degree of complexity in orientation mechanisms that we have.

The theory that otolithic cues provide a conflict signal limiting the expression of visually induced apparent body tilt is untenable for other reasons. The correct perception of body tilt cannot be obtained solely from a visual-canal interaction. Visual and oculomotor information alone is sufficient to specify the axis of relative disk-body rotation, but otolithic, proprioceptive, and somatosensory information is also necessary to determine the orientation of the disk surface and the axis of apparent body rotation in relation to the gravitational vertical. The paradoxical implication for the experiments of Held et al. (1975) and Young et al. (1975) is that gravity information is correctly interpreted in computing the orientation of the surface inducing illusory roll motion, but at the same time, it is inadequate to preserve correct perception of body attitude.

Our observations emphasize the contribution of information about gravity in a different fashion. All our experimental conditions included dynamic visual stimulation coupled with nonchanging otolithic and somatosensory stimulation. The visual stimulation was consistent only with physical body rotation around an axis normal to the disk surface and coincident with the line of sight. The absence of changing otolithic and somatosensory activity further restricted possible body motion in a terrestrial environment to rotation around a vertical axis. We propose that an internal representation of these constraints was taken into account when the perceptual system generated a response to the overall sensory context. This means that the absence of dynamic otolithic and somatosensory cues act in conjunction with the dynamic visual stimulation to constrain the character of experienced body motion and body configuration. Possible body motions, given the context of dynamic sensory stimulation in our conditions, included rotation around a vertical axis with the body in any orientation or configuration and with the disk horizontal.

Within the framework of these constraints, static cues about the particular configuration and orientation of the body and disk can be reinterpreted. For example, in our test situations in which the disk was tilted, perceptual remappings of static sensory stimuli occurred such that the disk would appear horizontal and the apparent line of sight would seem vertical even though the physical patterns of peripheral otolithic, somatosensory, and oculomotor stimulation were inconsistent with such a configuration. At other times, the apparent orientation of the head or torso with respect to gravity and the perceived configuration of head and torso were misapprehended. Such re- stricted and directed remappings show that the computations of body motion, configuration, and orientation must all have access to the same set of internal constraints and must be interrelated. This may also explain why only limited roll displacement from the upright was reported by the subjects of Held et al. (1975) and Young et al. (1975).

We did not specifically attempt to measure changes in the subjective vertical in the present study, although both Held et al. (1975) and Mauritz, Dichgans, and Hufschmidt (1977) have observed changes in the apparent visual vertical of subjects viewing a rotating visual display in the frontal plane. Our subjects reported their apparent orientation with respect to the subjective gravitational vertical. The perceptual remappings they experienced were due to alterations in the subjective vertical, apparent tilt of the body or disk, or changes in both, but the resultant patterns were always in accord with the constraints for body motion in a terrestrial environment.

The variability of the remappings occurring within our experimental conditions has significance for understanding mechanisms of spatial orientation. In Conditions B, $C$, and $D$, there were multiple patterns of experienced orientation reported across subjects (see Figure 4). Each of these patterns represents a compromise solution to the patterns of visual, otolithic, semicircular canal, proprioceptive, oculomotor, and somatosensory activity actually present. Each involves various perceptual remappings of static cues which restore concordance with the dynamic patterns of sensory information. However, for each pattern of dynamic stimulation present, there were many consistent perceptual remappings that could have occurred, and, in fact, many different ones were experienced. This is an example of one-to-many remapping, and is inconsistent with presently formulated convergence models of orientation.

In our test conditions, voluntary head or eye movements suppressed ISR and restored the sense of true body orientation and configuration. The suppressive effects of head movements are in accord with the earlier observations of Lackner and Texiera (1977) and Texiera and Lackner (1979), who found that both voluntary and passive swivel movements of the head suppressed ISR during incremental, stepwise exposure to optokinetic stimulation. The suppressive influence of eye movements demonstrates that varying visual and oculomotor signals, as well as vestibular ones, can attenuate the visual elicitation of apparent body motion. This means that dynamic covarying sensory inputs are highly important influences on spatial orientation. It should also be noted that no symptoms of motion sickness were elicited when head movements were made during illusory self-rotation; instead, the head movements suppressed illusory self-rotation.

From our findings we conclude that (1) dynamic stimulation has greater influence on apparent body orientation than does static stimulation, (2) the absence of synergistic patterns of expected input rival those patterns actually present in affecting experienced body orientation, con- 
figuration, and motion, and (3) the representation of body motion is not derived solely from primary motion detectors, but depends on virtually all receptor systems of the body, concurrent representations of overall body configuration and orientation, and a representation of what patterns are possible in a terrestrial environment.

\section{REFERENCES}

Allum, J. H. J., Graf, W., Dichgans, J., \& Schmidt, C. L. (1976). Visual-vestibular interactions in the vestibular nuclei of the goldfish. Experimental Brain Research, 26, 463-485.

Azzena, G. B., Azzena, M. T., \& Marini, R. (1974). Optokinetic nystagmus and the vestibular nuclei. Experimental Neurology, 42 , 158-168.

BLES, W. (1981). Circular vection and Coriolis effects. In J. Long \& A. Baddeley (Eds.), Attention and performance IX (pp. 47-61). Hillsdale, NJ: Erlbaum.

BLES, W., \& KAPTEYN, T. S. (1977). Circular vection and human posture. I. Does the proprioceptive system play a role? Agressologie, 18, 325-328.

Brandt, Th., Buchele, W., \& ARNold, F. (1977). Arthrokinetic nystagmus and egomotion sensation. Experimental Brain Research, 21, 331-338.

Daunton, N., \& ThOMSEn, D. (1979). Visual modulation of otolithdependent units in cat vestibular nuclei. Experimental Brain Research, 37, 173-176.

Dichgans, J., \& BRANDT, Th. (1972). Visual-vestibular interaction and motion perception. In J. Dichgans \& E. Bizzi (Eds.), Cerebral control of eye movements and motion perception (pp. 327-338). Basel and New York: Karger.

Dichgans, J., \& Brandt, Th. (1978). Visual-vestibular interaction: Effects on self-motion perception and postural control. In R. Held, H. Leibowitz, \& H.-L. Teuber (Eds.), Handbook of sensory physiology: Vol. 8. Perception (pp. 755-803). Berlin and New York: Springer-Verlag.

DoDGE, R. (1923). Thresholds of rotation. Journal of Experimental Psychology, 6, 107-137.

DuEnsing, F., \& Schaefer, K.-P. (1958). Die Aktivitat einzelner Neurone im Bereich der Vestibulariskerne bei Horizontalbeschleunigung under besonderer Berucksichtigung des vestibularen Nystagmus. $A r-$ chiv fïr Psychiatrie und Nervenkrankheiten, 198, 225-252.

Graybiel, A., \& PAtTerson, J. L. (1954). Thresholds of stimulation of the otolith organs as indicated by the oculogravic illusion (Research Report No. NM 001 059.01.38). Pensacola, FL: U.S. Naval School of Aviation Medicine.

GrUSSER, O.-J., \& GRUSSER-CORNEHLS, V. (1973). Neuronal mechanisms of visual movement perception and some psychophysical and behavioral correlations. In R. Jung (Ed.), Handbook of sensory physiology. Vol. 7, Part 3. Central processing of visual information (pp. 333-429). Berlin and New York: Springer-Verlag.

Held, R., Dichgans, J., \& BaUeR, J. (1975). Characteristics of moving visual scenes influencing spatial orientation. Vision Research, 15, 357-365.

Henn, V., Cohen, B., \& Young, L. (1980). Visual-vestibular interaction in motion perception and the generation of nystagmus. Neurosciences Research Program Bulletin, 18, 459-651.
Henn, V., Young, L. R., Finley, C. (1974). Vestibular nucleus units in alert monkeys are also influenced by moving visual field. Brain Research, 71, 144-149.

HUANG, J. K., \& YoUNG, L. R. (1981). Sensation of rotation about a vertical axis with a fixed visual field in different illuminations and in the dark. Experimental Brain Research, 41, 172-183.

LACKNER, J. R. (1977). Induction of nystagmus in stationary subjects with a rotating sound field. Aviation, Space \& Environmental Medicine, 48, 501-502.

LACKNER, J. R., \& DiZıo, P. A. (1984). Some efferent and somatosensory influences on body orientation and oculomotor control. In R. Wooten \& L. Spillmann (Eds.), Sensory experience, adaptation, and perception (pp. 281-301). Hillsdale, NJ: Erlbaum.

LACKNER, J. R., \& LEVINE, M. L. (1979). Changes in apparent body orientation and sensory localization induced by vibration of postural muscles: Vibratory myesthetic illusions. Aviation, Space \& Environmental Medicine, 50, 346-354.

LACKNER, J. R., \& TEXIERA, R. (1977). Visual-vestibular interaction: Vestibular stimulation suppresses the visual induction of illusory selfrotation. Aviation, Space \& Environmental Medicine, 48, 248-253.

MACH, E. (1959). The analysis of sensations (S. Waterlow, Trans.). New York: Dover. (5th German ed. originally published 1906).

Mauritz, K. H., Dichgans, J., \& Hufschmidt, A. (1977). The angle of visual roll motion determines displacement of subjective visual vertical. Perception \& Psychophysics, 22, 557-562.

Mergner, T., NARd, G. L., Becker, W., \& DeEcke, L. (1983). The role of canal-neck interaction for the perception of horizontal trunk and head rotation. Experimental Brain Research, 49, 198-208.

Rubin, A. M., Liedgren, S. R., Milne, A. L., Young, J. A., \& FREDRICKSON, J. M. (1977). Vestibular and somatosensory interaction on the cat vestibular nuclei. Pflügers Archiv, 371, 155-160.

TEXIERA, R., \& LACKNeR, J. R. (1979). Optokinetic motion sickness: Attenuation of visually induced apparent self-rotation by passive head movements. Aviation, Space \& Environmental Medicine, 50, 264-266.

Thoden, V., Golsong, R., \& WIRBITZKY, J. (1975). Cervical influence on single units of vestibular and reticular nuclei in cats. Pflügers Archiv, 355, R101.

WAESPE, W., \& HENN, V. (1977). Neuronal activity in the vestibular nuclei of the alert monkey during vestibular and optokinetic stimulation. Experimental Brain Research, 27, 523-538.

WAESPE, W., WAESPE, B., \& HENN, V. (1980). Subjective velocity estimation during visual-vestibular conflict. Archives of Psychiatry, 228, 109-116.

Wilson, V. J., Kato, M., Thomas, R. C., \& Peterson, B. W. (1966). Excitation of lateral vestibular neurons by peripheral afferent fibers. Journal of Neurophysiology, 29, 508-529.

Young, L. R., Dichgans, J., Murphy, R., \& Brandt, Th. (1973). Interaction of optokinetic and vestibular stimuli in motor perception. Acta Oto-Laryngologica, 76, 24-31.

Young, L. R., Oman, C. M., \& Dichgans, J. M. (1975). Influence of head orientation on visually induced pitch and roll sensation. $A v i$ ation, Space \& Environmental Medicine, 45, 264-268.

ZaCharJas, G. L., \& YounG, L. R. (1981). Influence of combined visual and vestibular cues on human perception and control of horizontal rotation. Experimental Brain Research, 41, 159-171.

(Manuscript received February 26, 1985 revision accepted for publication October 28, 1985.) 